\title{
Analysis of Coupled Exponential Microstrip Lines by Means of a Multi-Step Perturbation Technique
}

\author{
Paolo Manfredi, Daniël De Zutter, and Dries Vande Ginste \\ IBCN/Electromagnetics Group, Department of Information Technology, Ghent University/iMinds \\ Sint-Pietersnieuwstraat 41, 9000 Gent, Belgium \\ E-mail: paolo.manfredi@ugent.be
}

\begin{abstract}
In this contribution, an iterative and adaptive multistep perturbation technique for nonuniform transmission lines is presented and applied to the analysis of coupled exponential lines. The Telegrapher's equations for nonuniform lines, which do not have a closed-form solution, are recast as the equations for uniform lines with equivalent distributed sources, for which a well-known numerical solution procedure exists. The line voltages and currents are computed in multiple steps by iteratively updating the distributed sources. The method turns out to be faster than classical solutions based on the discretization of the line into uniform subsections. Two validation examples are proposed that deal with coupled exponential lines, which have relevant applicability in microwave components.
\end{abstract}

Index Terms-Exponential lines, nonuniform transmission lines, perturbation methods, transmission line theory.

\section{INTRODUCTION}

Nonuniform transmission lines (NUTL) have wide applicability in the microwave field as impedance transformers, filters, or directional couplers, just to mention a few [1]. In particular, exponential lines are an important category of NUTLs and their analysis has been extensively studied [2], [3].

In general, however, no exact solution exists for NUTLs. Perhaps the most common and natural approach is to study a NUTL as a cascade connection of many locally uniform sections [4], [5]. Alternative approaches were proposed but with some limitations in terms of assumptions or ease of implementation [6]-[12].

A two-step perturbation technique was proposed as an accurate and more general approach [13] and applied to the analysis of the effects of fiber weave [14] and line-edge roughness [15]. The nonuniformity is interpreted as a perturbation of a uniform line with averaged per-unit-length (p.u.l.) parameters. This formulation results in the contribution of the place-dependent part of the p.u.l. parameters to appear under the form of equivalent distributed sources. Semi-analytical expressions were derived for the corresponding first- and second-order perturbations of the line voltages and currents. However, the calculation of only two perturbations does not always provide enough accuracy. The discretization of the line into several nonuniform subsections was proposed to circumvent this problem at the expense of a substantial efficiency reduction [14].

In order to remedy this drawback, an iterative reformulation of this method has been recently proposed [16], allowing to readily calculate an arbitrary number of perturbation terms and adaptively stop the solution when convergence within a given threshold is achieved. The solution of the equivalent problem with distributed sources is calculated in analogy with an external field illumination [4]. Whereas originally applied to cable bundles, the technique is here applied to the analysis of coupled exponential on-board lines. Two validation examples taken from the literature are provided.

\section{Multi-Step Perturbation Technique for NUTL}

A coupled NUTL is described in the frequency-domain by Telegrapher's equations with place-dependent p.u.l. parameters

$$
\begin{gathered}
\frac{d}{d z} \boldsymbol{V}(z)=-j \omega \boldsymbol{L}(z) \boldsymbol{I}(z) \\
\frac{d}{d z} \boldsymbol{I}(z)=-j \omega \boldsymbol{C}(z) \boldsymbol{V}(z)
\end{gathered}
$$

where $z \in[0, \ell]$ denotes the longitudinal coordinate, vectors $\boldsymbol{V}=\left[V_{1}, V_{2}\right]^{T}$ and $\boldsymbol{I}=\left[I_{1}, I_{2}\right]^{T}$ collect the voltages and currents along the line, and $\boldsymbol{L}$ and $\boldsymbol{C}$ are the $2 \times 2$ p.u.l. inductance and capacitance matrices. Losses are readily included as the imaginary part of these matrices.

The starting point of the proposed perturbation technique is to express the place-dependent p.u.l. parameters in terms of a constant (i.e., place-independent) component plus a placedependent variation:

$$
\begin{aligned}
\boldsymbol{L}(z) & =\tilde{\boldsymbol{L}}+\Delta \boldsymbol{L}(z) \\
\boldsymbol{C}(z) & =\tilde{\boldsymbol{C}}+\Delta \boldsymbol{C}(z)
\end{aligned}
$$

The constant components are calculated by averaging along the interconnect length, i.e.

$$
\begin{aligned}
\tilde{\boldsymbol{L}} & =\frac{1}{\ell} \int_{0}^{\ell} \boldsymbol{L}(z) d z \\
\tilde{\boldsymbol{C}} & =\frac{1}{\ell} \int_{0}^{\ell} \boldsymbol{C}(z) d z
\end{aligned}
$$

Next, the line voltages and currents are expressed in terms of multiple perturbations

$$
\begin{gathered}
\tilde{\boldsymbol{V}}(z)=\boldsymbol{V}_{0}(z)+\sum_{k=1}^{K} \boldsymbol{V}_{k}(z) \\
\tilde{\boldsymbol{I}}(z)=\boldsymbol{I}_{0}(z)+\sum_{k=1}^{K} \boldsymbol{I}_{k}(z)
\end{gathered}
$$


up to an arbitrary order $K$. Substituting (2) and (4) into (1) and collecting the terms of the same perturbation order leads to

$$
\begin{aligned}
\frac{d}{d z} \boldsymbol{V}_{k}(z) & =-j \omega \tilde{\boldsymbol{L}} \boldsymbol{I}_{k}(z)-j \omega \Delta \boldsymbol{L}(z) \boldsymbol{I}_{k-1}(z) \\
\frac{d}{d z} \boldsymbol{I}_{k}(z) & =-j \omega \tilde{\boldsymbol{C}} \boldsymbol{V}_{k}(z)-j \omega \Delta \boldsymbol{C}(z) \boldsymbol{V}_{k-1}(z)
\end{aligned}
$$

for $k=0, \ldots, K$. The above equations govern the $k$ th perturbation term of the line voltages and currents. It is worth noting that 1) the first terms in the r.h.s. of (5) describe a uniform transmission line with place-independent p.u.l. parameters $\tilde{\boldsymbol{L}}$ and $\tilde{\boldsymbol{C}}$, and 2) the remaining terms play the role of distributed sources. The distributed sources account for the place-dependent component of the p.u.l. parameters and depend on the $(k-1)$ th perturbation term. A solution for equations of the form (5) is available and it is traditionally used to account for the effect of an external field impinging on a uniform transmission line [4]. A similar procedure can be used to iteratively solve (5) for increasing perturbation order $k$, starting from the solution of the unperturbed line for $k=0$ and with $\boldsymbol{V}_{-1}=\boldsymbol{I}_{-1}=0$. The iterations are stopped when the solution has converged (e.g., when the new perturbation terms become sufficiently small). Intuitively, the series (4) is expected to converge as long as the longitudinal variations $\Delta \boldsymbol{L}$ and $\Delta C$ are reasonably "small", with a descreasing rate for larger perturbations (see the application examples). However, no rigorous proof as to the guaranteed convergence of (4) could be found so far.

The explicit solution of (5) reads

$$
\begin{gathered}
\boldsymbol{V}_{k}(z)=\boldsymbol{\Phi}_{11}(z) \boldsymbol{V}_{k}(0)+\boldsymbol{\Phi}_{12}(z) \boldsymbol{I}_{k}(0)+\boldsymbol{V}_{F T, k}(z) \\
\boldsymbol{I}_{k}(z)=\boldsymbol{\Phi}_{21}(z) \boldsymbol{V}_{k}(0)+\boldsymbol{\Phi}_{22}(z) \boldsymbol{I}_{k}(0)+\boldsymbol{I}_{F T, k}(z)
\end{gathered}
$$

where

$$
\left[\begin{array}{ll}
\boldsymbol{\Phi}_{11}(z) & \boldsymbol{\Phi}_{12}(z) \\
\boldsymbol{\Phi}_{21}(z) & \boldsymbol{\Phi}_{22}(z)
\end{array}\right]=\exp \left(-j \omega\left[\begin{array}{cc}
0 & \tilde{\boldsymbol{L}} \\
\tilde{\boldsymbol{C}} & 0
\end{array}\right] z\right)
$$

and

$$
\begin{aligned}
\boldsymbol{V}_{F T, k}(z)= & -j \omega \int_{0}^{z} \boldsymbol{\Phi}_{11}(z-\tau) \Delta \boldsymbol{L}(\tau) \boldsymbol{I}_{k-1}(\tau) d \tau \\
& -j \omega \int_{0}^{z} \boldsymbol{\Phi}_{12}(z-\tau) \Delta \boldsymbol{C}(\tau) \boldsymbol{V}_{k-1}(\tau) d \tau \\
\boldsymbol{I}_{F T, k}(z)= & -j \omega \int_{0}^{z} \boldsymbol{\Phi}_{21}(z-\tau) \Delta \boldsymbol{L}(\tau) \boldsymbol{I}_{k-1}(\tau) d \tau \\
& -j \omega \int_{0}^{z} \boldsymbol{\Phi}_{22}(z-\tau) \Delta \boldsymbol{C}(\tau) \boldsymbol{V}_{k-1}(\tau) d \tau
\end{aligned}
$$

The voltages $\boldsymbol{V}_{k}(0)$ and the currents $\boldsymbol{I}_{k}(0)$ at the line origin are obtained by incorporating the information on the line terminations. For additional information on the general solution procedure, the reader is referred to [16].

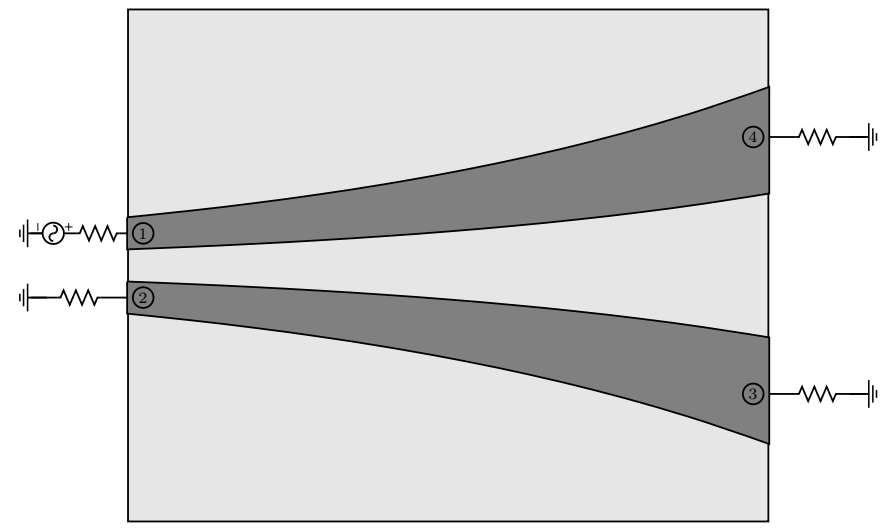

Fig. 1. Top view of a coupled exponential line with port definition.

\section{APPLICATION EXAMPLE: EXPONENTIAL LINES}

The technique has been implemented in MATLAB and it is applied to the analysis of coupled exponential lines, whose layout is sketched in Fig. 1.

In the first example, the NUTL is defined by the following place-dependent p.u.l. inductance and capacitance matrices [12]:

$$
\begin{aligned}
& \boldsymbol{L}(z)=\boldsymbol{L}_{0} e^{q z / \ell} \\
& \boldsymbol{C}(z)=\boldsymbol{C}_{0} e^{-q z / \ell}
\end{aligned}
$$

with

$$
\begin{aligned}
\boldsymbol{L}_{0} & =\left[\begin{array}{rr}
171.4 & 18.65 \\
18.65 & 171.4
\end{array}\right] \mathrm{nH} / \mathrm{m} \\
\boldsymbol{C}_{0} & =\left[\begin{array}{rr}
65.7 & -7.15 \\
-7.15 & 65.7
\end{array}\right] \mathrm{pF} / \mathrm{m}
\end{aligned}
$$

Results are computed at $1 \mathrm{GHz}$ for a line with $q=1$ and length $\ell=20 \mathrm{~cm}$. The first line is excited with a $1 \mathrm{~V}$ voltage source. The termination impedances for both lines are $50 \Omega$ at $z=0$ and $50 \exp (q)=136 \Omega$ at $z=\ell$. The largest deviation of the p.u.l. inductance and capacitance matrices from their average values occurs at $z=\ell$ and $z=0$, respectively, and amounts to $58.2 \%$ in norm.

Fig. 2 shows the magnitude (top panel) and phase (bottom panel) of the voltages along the two conductors of the line. The blue lines provide a reference computed by discretizing the line into 1001 uniform sections. The dashed black line is the result obtained with the perturbation technique with two steps only $(K=2)$, as from the previously proposed framework in [13]-[15]. There is an appreciable lack of accuracy, especially on the magnitude of the voltage along the active conductor and on the phases of both voltages. Finally, the dashed red line is the result computed with the proposed iterative multi-step perturbation technique, which shows excellent agreement. The iterative solution is stopped after the perturbation terms have become smaller in magnitude than $1 \%$ of the achieved solution. This leads the algorithm to compute up to 11 perturbation terms. 

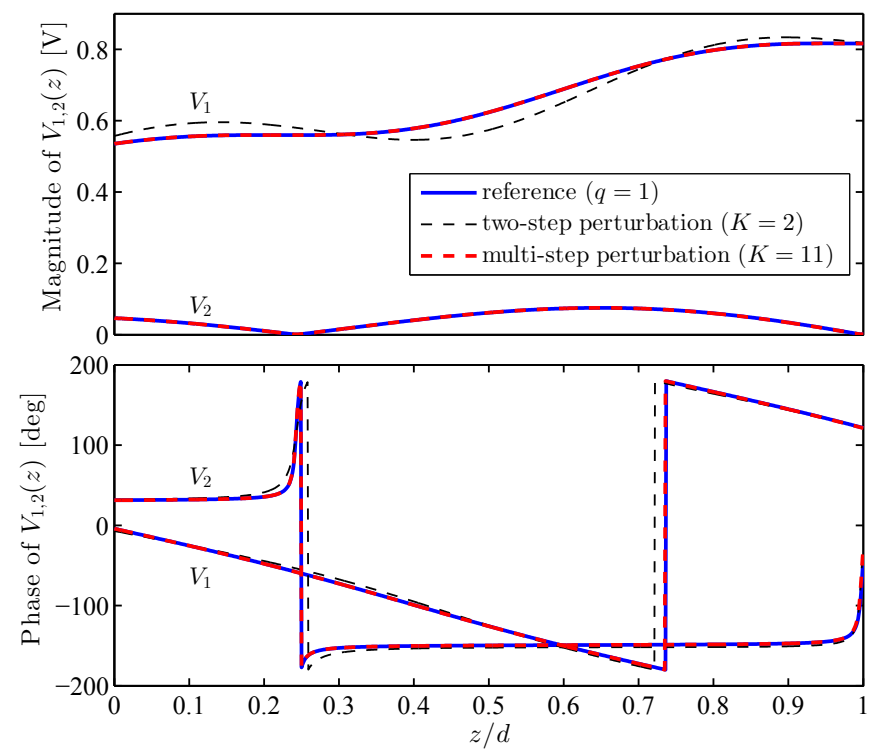

Fig. 2. Magnitude and phase of the voltages along the exponential line with $q=1$ at $1 \mathrm{GHz}$. The solid blue line is the reference result, while the dashed black and red lines are the results of the previous two-step [13] and the new multi-step perturbation techniques, respectively.

In order to assess the effect of larger longitudinal variations, Fig. 3 provides the results computed for a line with $q=2$ (load impedance: $369 \Omega$ ). In this case, the maximum variation of the p.u.l. inductance and capacitance matrices is $131.2 \%$ in norm. Despite this very large variation, the perturbation technique still converges although, as expected, it requires more iterations, i.e., 65. Moreover, the solution for $K=2$ turns out to be way less accurate than in the previous case.
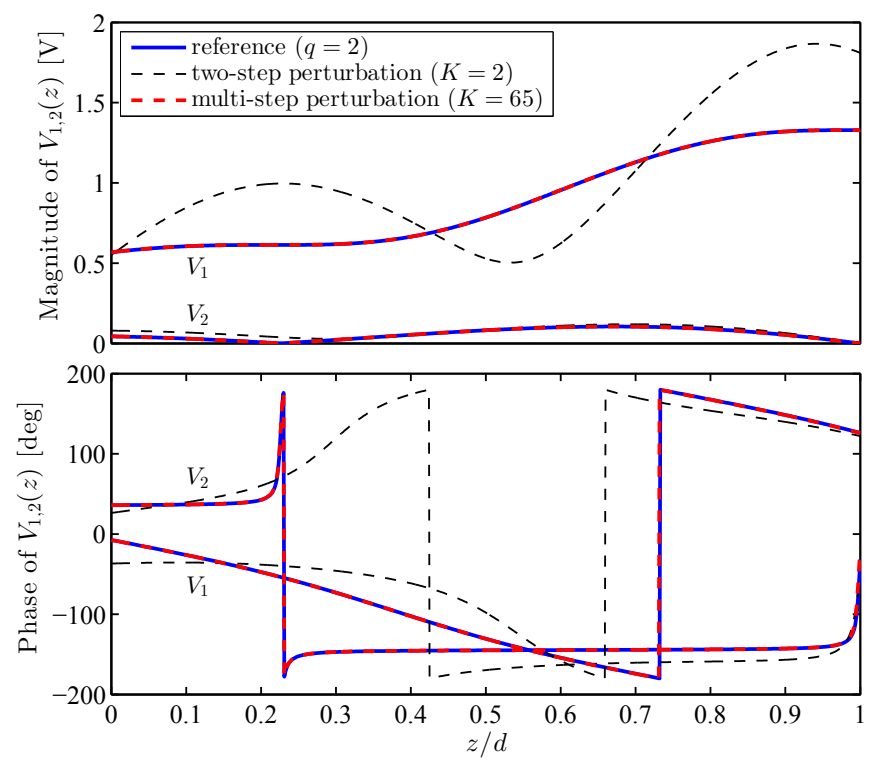

Fig. 3. Magnitude and phase of the voltages along the exponential line with $q=2$ at $1 \mathrm{GHz}$. Curve identification is as in Fig. 2.

The second example refers to a coupled exponential line designed for use as a directional coupler [3]. In this case, the geometry of the microstrip lines is designed so that the even and odd mode characteristic impedances exhibit exponential behavior:

$$
\begin{aligned}
& Z_{e}(z)=Z_{e, 0} e^{-\alpha z} \\
& Z_{o}(z)=Z_{o, 0} e^{+\alpha z}
\end{aligned}
$$

with $Z_{e, 0}=81.4 \Omega, Z_{o, 0}=29.73 \Omega$, and $\alpha=40 \mathrm{~m}^{-1}$. The traces have a length $\ell=1.25 \mathrm{~cm}$ and are fabricated on an alumina substrate with a thickness of $0.635 \mathrm{~mm}$ and a relative permittivity of 9.7 . The trace width and separation are computed at discrete locations to achieve the target values of the characteristic impedances (9). The resulting phase velocities $v_{e}$ and $v_{o}$ of the even and odd modes, as reported in [3], are provided in Table I. For the simulation performed in the following, the even and odd mode parameters are converted into the standard p.u.l. inductance and capacitance matrices. Owing to the small longitudinal variation of the phase velocities, a linear interpolation of the values in Table I is used to retrieve the values at an arbitrary position $z$. For this line, the largest longitudinal deviations of the p.u.l. inductance and capacitance matrix are $26.6 \%$ and $24.5 \%$ in norm, respectively.

Fig. 4 shows the $\mathrm{S}$-parameters of the direction coupler. The reference results (solid blue lines) are again computed by discretizing the line into 1001 uniform sections. The results obtained with the two-step perturbation technique, although good for some S-parameters and at the lower frequencies, show a substantial discrepancy at high frequencies, in particular for $S_{11}$. In order to accurately capture the behavior of $S_{11}$, the relative threshold for stopping the iterative solution is set to $0.01 \%$ of the computed voltage and current values. As a result, the number of calculated perturbation steps ranges from 3 to a maximum of 8 , depending on the frequency. The calculation of the reference solution takes $12.9 \mathrm{~s}$, as opposed to $3.1 \mathrm{~s}$ required by the proposed multi-step perturbation technique, which is therefore more than 4 times faster.

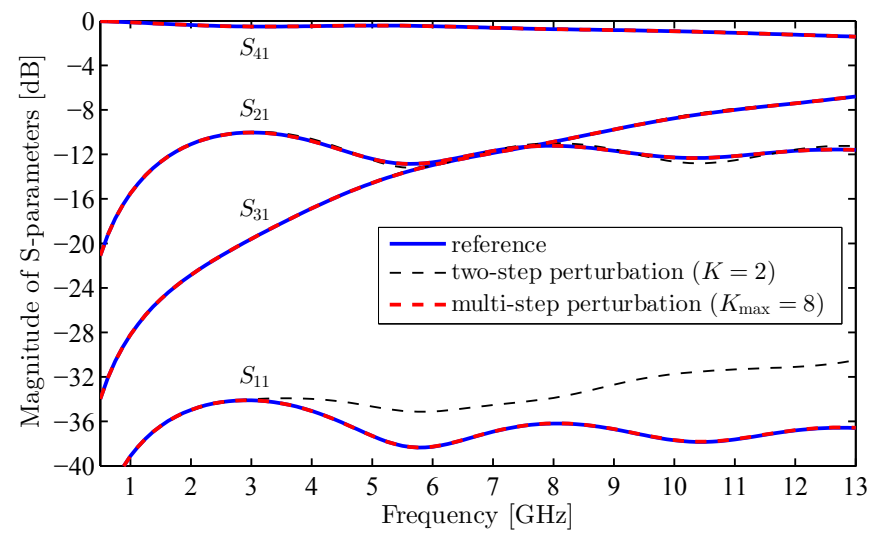

Fig. 4. Magnitude of the S-parameters of the directional coupler. Curve identification is as in Fig. 2. 
TABLE I

PHASE VELOCITIES OF EVEN AND ODD MODES AT DISCRETE LONGITUDINAL LOCATIONS.

\begin{tabular}{|c||c|c|c|c|c|c|c|c|c|c|c|}
\hline$z / \ell$ & 0.0 & 0.1 & 0.2 & 0.3 & 0.4 & 0.5 & 0.6 & 0.7 & 0.8 & 0.9 & 1.0 \\
\hline \hline$v_{e} \times 10^{-9} \mathrm{~m} / \mathrm{s}$ & 0.114 & 0.113 & 0.113 & 0.113 & 0.113 & 0.113 & 0.114 & 0.114 & 0.114 & 0.115 & 0.116 \\
\hline$v_{o} \times 10^{-9} \mathrm{~m} / \mathrm{s}$ & 0.131 & 0.131 & 0.131 & 0.131 & 0.130 & 0.129 & 0.128 & 0.127 & 0.124 & 0.123 & 0.120 \\
\hline
\end{tabular}

\section{CONCLUSIONS}

This paper presents an iterative and adaptive multi-step perturbation technique for the analysis of arbitrary NUTLs. By considering the longitudinal variation of the p.u.l. parameters as a perturbation with respect to an average value, the corresponding perturbations of the line voltages and currents are iteratively computed as the solution of a uniform transmission line with equivalent distributed sources. The technique is applied to the analysis of exponential lines and it is shown to be very accurate and computationally efficient. The convergence rate turns out to be slower or faster depending on the amount of longitudinal variation of the p.u.l. inductance and capacitance matrices.

\section{ACKNOWLEDGMENT}

This work was supported by the Research Foundation Flanders (FWO-Vlaanderen), of which Paolo Manfredi is a Post-Doctoral Research Fellow.

\section{REFERENCES}

[1] S. Yamamoto, T. Azakami, and K. Itakura, "Coupled nonuniform transmission line and its applications," IEEE Trans. Microw. Theory Techn., vol. 15 , no. 4 , pp. 220-231, Apr. 1967.

[2] C. P. Womack, "The use of exponential transmission lines in microwave components," IEEE Trans. Microw. Theory Techn., vol. 10, no. 2, pp. 124 132, Mar. 1962.

[3] M. I. Sobhy and E. A. Hosny, "The design of directional couplers using exponential lines in inhomogeneous media," IEEE Trans. Microw. Theory Techn., vol. 30, no. 1, pp. 71-76, Jan. 1982.

[4] C. R. Paul, Analysis of Multiconductor Transmission Lines, New York: Wiley, 1994.
[5] J.-F. Mao and Z.-F. Li, "Analysis of the time response of nonuniform multiconductor transmission lines with a method of equivalent cascaded network chain," IEEE Trans. Microw. Theory Techn., vol. 40, no. 5, pp. 948-954, May 1992.

[6] G.-W. Pan, G. J. Wunsch, and B. K. Gilbert, "Frequency-domain analysis of coupled nonuniform transmission lines using Chebyshev pseudospatial techniques," IEEE Trans. Microw. Theory Techn., vol. 40, no. 11, pp. 2025-2033, Nov. 1992

[7] F.-Y. Chang, "Waveform relaxation analysis of nonuniform lossy transmission lines characterized with frequency-dependent parameters," IEEE Trans. Circuits Syst., vol. 38, no. 12, pp. 1484-1500, Dec. 1991.

[8] D. Kuznetsov, "Efficient circuit simulation of nonuniform transmission lines," IEEE Trans. Microw. Theory Techn., vol. 46, no. 5, pp. 546-550, May 1998.

[9] S. Grivet-Talocia and F. Canavero, "Wavelet-based adaptive solution for the nonuniform multiconductor transmission lines," IEEE Microw. Guid. Wave Lett., vol. 8, no. 8, pp. 287-289, Aug. 1998.

[10] Q. Xu and P. Mazumder, "Accurate modeling of lossy nonuniform transmission lines by using differential quadrature methods," IEEE Trans. Microw. Theory Techn., vol. 50, no. 10, pp. 2233-2246, Oct. 2002.

[11] E. Gad and M. Nakhla, "Efficient simulation of nonuniform transmission lines using integrated congruence transform," IEEE Trans. Very Large Scale Integr. (VLSI) Syst., vol. 12, no. 12, pp. 1307-1320, Dec. 2004.

[12] M. Khalaj-Amirhosseini, "Analysis of coupled nonuniform transmission lines using Taylor's series expansion," IEEE Trans. Electromagn. Compat., vol. 48, no. 8, pp. 594-600, Aug. 2006.

[13] M. Chernobryvko, D. De Zutter, and D. Vande Ginste, "Nonuniform multiconductor transmission line analysis by a two-step perturbation technique," IEEE Trans. Compon. Packag. Manuf. Techol., vol. 4, no. 11, pp. 1838-1846 , Nov. 2014

[14] M. Chernobryvko, D. De Zutter, and D. Vande Ginste, "A perturbation technique to analyze the influence of fiber weave effects on differential signaling," in Proc. IEEE 20th Conf. Elect. Perform. Electron. Packag. Syst., San Jose, CA, Oct. 2013, pp. 15-18.

[15] P. Manfredi, D. Vande Ginste, and D. De Zutter, "An effective modeling framework for the analysis of interconnects subject to line-edge roughness," IEEE Microw. Wireless Compon. Lett., vol. 25, no. 8, pp. 502-504, Aug. 2015.

[16] P. Manfredi, D. De Zutter, and D. Vande Ginste, "Analysis of nonuniform transmission lines with an iterative and adaptive perturbation technique," IEEE Trans. Electromagn. Compat., DOI: 10.1109/TEMC.2016.2523604. 